POS $\quad$ PROCEEDINGS

\title{
Self-Gravitating Warped Disks Around Black Holes
}

\author{
Ayse Ulubay-Siddiki \\ Max Planck Institute for Extraterrestrial Physics, Giessenbachstrasse, D-85748 Garching, \\ Germany \\ E-mail: siddiki@mpe.mpg.de

\section{Ortwin Gerhard} \\ Max Planck Institute for Extraterrestrial Physics, Giessenbachstrasse, D-85748 Garching, \\ Germany \\ E-mail: gerhard@mpe.mpg.de

\section{Magda Arnaboldi} \\ European Southern Observatory, Karl-Schwarzschild-Strasse 2, D-85748 Garching, Germany \\ E-mail: marnaboleeso.org
}

\begin{abstract}
We address the problem of finding steadily precessing nonlinear warped disk solutions for disks around black holes. The disk is modeled as a collection of concentric, circular rings surrounding a black hole. In our model, the rings interact with each other gravitationally, which causes precession of the ring orbits around the direction of the total disk angular momentum. When the torque around the line-of-nodes, i.e. where all the rings intersect, is zero, equilibrium inclinations can be obtained fixing a common precession frequency. We show that there exists a scaling relation between the parameters of the disk, and the black hole mass for a given warp shape. We find that for a range of parameters, the disk might get highly warped, and obscure the central radiation source. We also analyze the stability of such equilibrium solutions by perturbation theory, and time integration of equations of motion.
\end{abstract}

VII Microquasar Workshop: Microquasars and Beyond September 1 - 5, 2008

Foca, Izmir, Turkey 


\section{Introduction}

Warped disks where the inclination of a disk with respect to a preferred plane changes gradually with radius are common in astrophysics. On galactic scales, warping in the nuclear disks surrounding supermassive black holes is hinted by the observations of maser sources. NGC4258 is the first galaxy shown to host such a disk in its nucleus [9,5]. Today, many other nuclei are known to have such warped disks $[4,3]$. Among the many mechanisms by which the disk might be excited to become warped, radiation pressure of a central source, and the interaction of a tilted disk with a rotating black hole (the Bardeen-Petterson effect), are the most widely studied ones $[11,8,10,7]$.

The goal of this study is to investigate the possibility of steadily precessing, stable, nonlinearly warped self-gravitating disks in the (Keplerian) gravitational potential of a black hole. Assuming that the warp has been excited by either of the mechanisms listed above, we aim to answer the question of whether the disk self-gravity can maintain the warped shape even after the exciting torque has ceased to exist.

\section{The Model}

We consider a cold disk where the disk elements move on very nearly circular orbits. Following similar models of Galactic warps (e.g. [1,6]) we model such a disk as a collection of concentric circular rings with mass $m$, and radius $r$, which are tilted with respect to each other. Geometry of the rings is defined through the Euler angles $(\theta, \phi, \psi)$. The rings orbit around a central black hole with angular momentum $p_{\psi}=m r^{2} \Omega$, while they precess around the total angular momentum direction with a frequency $\dot{\phi}$ due to the self-gravity torques. In this case, the equations of motion for rings read:

$$
\begin{aligned}
& p_{\theta_{i}}=\frac{m_{i} r_{i}^{2}}{2} \dot{\theta}_{i} \\
& p_{\phi_{i}}=\frac{m_{i} r_{i}^{2}}{2} \dot{\phi}_{i} \sin ^{2} \theta_{i}+p_{\psi_{i}} \cos \theta_{i}, \\
& \dot{p_{\theta_{i}}}=\frac{m_{i} r_{i}^{2}}{2} \dot{\phi}_{i}^{2} \sin \theta_{i} \cos \theta_{i}-\dot{\phi}_{i} p_{\psi_{i}} \sin \theta_{i}-\frac{\partial V\left(r_{i}, \theta_{i}, \phi_{i}\right)}{\partial \theta_{i}}, \\
& \dot{p}_{\phi_{i}}=-\frac{\partial V\left(r_{i}, \theta_{i}, \phi_{i}\right)}{\partial \phi_{i}}
\end{aligned}
$$

where $p_{\phi}$ is around the z-axis, and $p_{\theta}$ is around the line-of-nodes(LONs). The potential energy $V\left(r_{i}, \theta_{i}, \phi_{i}\right)$ has two components; one arises due to the central black hole, and the other one due to the interaction of the ring under consideration with all the other rings. In evaluating the latter, we use an implementation of the code developed by [1] originally for polar ring galaxies.

\section{Steadily Precessing Equilibria}

Equilibrium for a system of rings as described above is defined such that the rings precess on a common LONs, without changing their inclination (i.e. $\dot{\theta}=0$ ). For a given precession frequency, equilibrium inclinations can be obtained by solving equation (2.3) simultaneously for all the rings. Figure 1 shows three example disk solutions for different disk-to-black hole mass fractions. The 


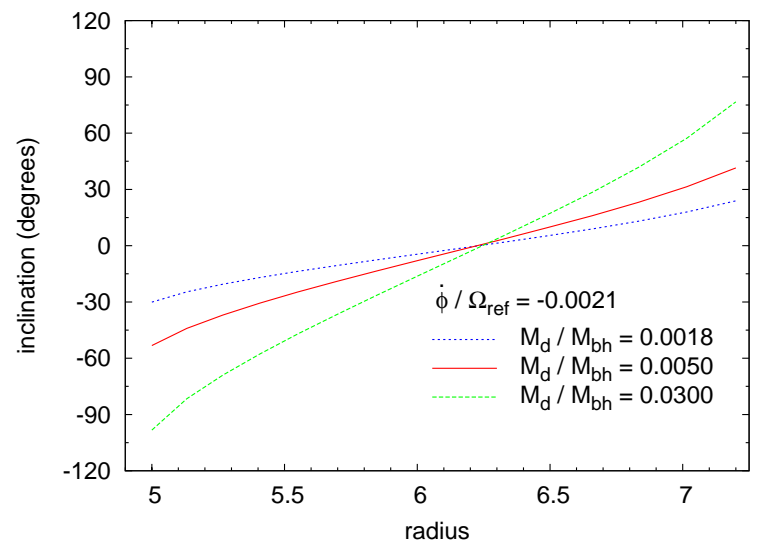

Figure 1: Example warped equilibria for three different disk-to-black hole mass fractions.

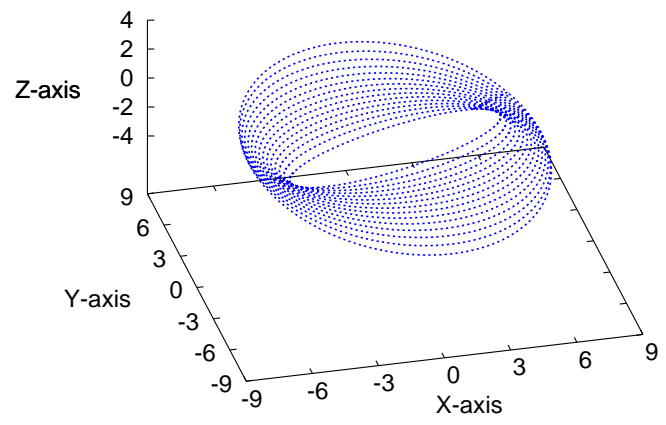

Figure 2: 3D view of the disk corresponding to the lowest mass case shown in the above plot.

radial extents, and the normalized precession frequencies of these disks are identical. As one sees, an increase in the disk mass results in a more highly inclined warp equilibrium. In figure 2 we show the 3-dimensional view of the disk corresponding to the lowest mass case shown in figure 1.

\subsection{Scaling the Solutions}

The torque between any of the two rings $(i, j)$ with a mutual inclination $\alpha_{i j}$ has the following dependency:

$$
\frac{\partial V_{i j}}{\partial \alpha_{i j}} \propto \frac{m_{i} m_{j} r_{i} r_{j} \sin 2 \alpha_{i j}}{\left(r_{i}^{2}+r_{j}^{2}\right)^{3 / 2}}
$$

We express all the ring masses and radii in terms of the mass and radius of a reference ring, i.e. $\mu_{i} \equiv m_{i} / m_{r e f}, v_{i} \equiv r_{i} / r_{r e f}$, and similar for $j$. We insert this into equation (3.1), and then insert the resulting equation into equation (2.3). Normalizing the precession frequency to the orbital frequency of the reference ring we arrive at:

$$
\frac{\dot{\phi}}{\Omega_{r e f}} \simeq C\left(\theta_{i}\right) \frac{m_{r e f}}{M_{b h}}
$$


after some algebra, where $C\left(\theta_{i}\right)$ is a constant. From equation (3.2) it follows that the precessing equilibrium remains unchanged in shape for those variations of $\left(\dot{\phi}, \Omega_{r e f}, m_{r e f}, M_{b h}\right)$ that keep the ratio $\left(\dot{\phi} / \Omega_{r e f}\right) /\left(m_{r e f} / M_{b h}\right)$ constant.

\subsection{Stability of the Disk}

After evaluating the equilibrium configurations, we go on analyzing their stability via two methods. First, we apply perturbation theory to the equations of motion, and look for stable solutions such that small deviations from an equilibrium position do not grow exponentially. Second, we perform a time integration of the equations of motion. In this case, the stability of the disk is defined such that the rings continue precessing on a common LONs throughout their evolution. In figures 3, and 4 we show examples of the time evolution analysis. The symbols starting from the outer circles show how the inclination changes with radius as shown on the horizontal scale. The symbols starting from the inner circle show how the azimuthal angle $\phi$ changes with the ring radius; for this part of the plot ring radii are scaled to half of their values to make the figures more easy to read.
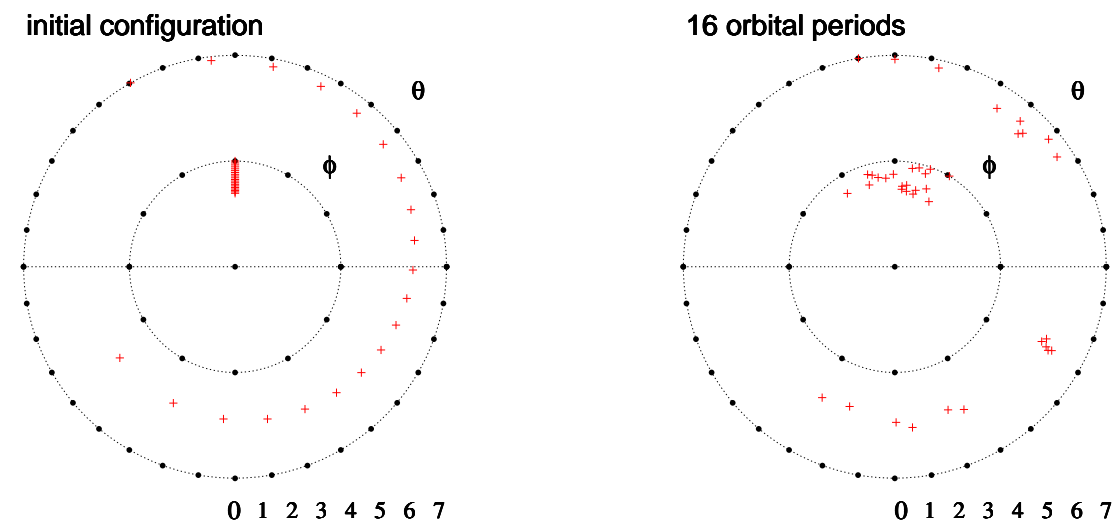

Figure 3: Time evolution of a disk with $M_{d} / M_{b h}=0.1, r_{\text {out }} / r_{\text {in }}=1.44$, and $\dot{\phi} / \Omega_{\text {ref }}=-0.0021$. The disk breaks up into parts after a few orbital periods, hence it is unstable.

\section{Summary and Conclusions}

In this study we have investigated warped disk solutions around black holes for which the only acting force is gravity. We used a simple model where the disk is a collection of concentric circular nested rings. We have shown that with these approximations, steadily precessing disks can be constructed. Stability was determined by perturbation theory, and by direct time integration.

The disks we have studied obey a scaling relation: they can be scaled to arbitrary radii $\mathrm{r}$, given that the precession frequency is normalized to the orbital frequency, and they can be scaled in mass, given that the ratio of precession frequency to the orbital frequency is changed proportional to the disk-to-black hole mas ratio. 

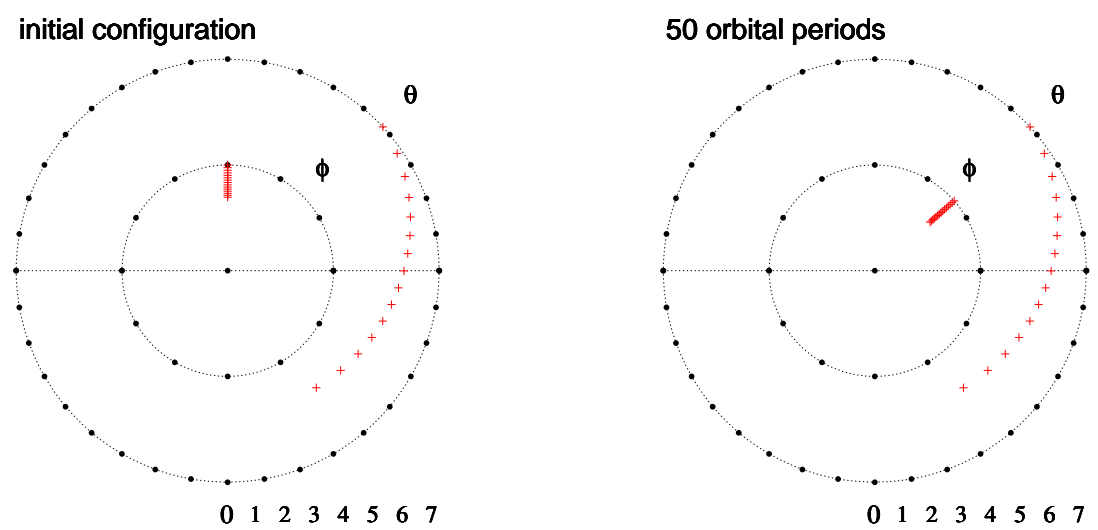

Figure 4: Time evolution of a disk with $M_{d} / M_{b h}=0.005, r_{\text {out }} / r_{\text {in }}=1.44$, and $\dot{\phi} / \Omega_{\text {ref }}=-0.0021$. The rings precess on a common LONs for many orbital periods, hence we judge the disk is stable.

\section{References}

[1] Arnaboldi, M., Sparke, L. S., AJ, Self-gravitating polar rings in axisymmetric and triaxial galaxy potentials, 107, 958 (1994)

[2] Bardeen, J. M., Petterson, J. A., ApJL, The Lense-Thirring Effect and Accretion Disks around Kerr Black Holes, 195, L65 (1975)

[3] Gallimore, J. F., Baum, S. A., O’Dea, C. P., ApJ, The Parsec-Scale Radio Structure of NGC 1068 and the Nature of the Nuclear Radio Source, 613, 794 (2004)

[4] Greenhill, L. J., Booth, R. S., Ellingsen, S. P., Herrnstein, J. R., Jauncey, D. L., McCulloch, P. M., Moran, J. M., Norris, R. P., Reynolds, J. E., Tzioumis, A. K., ApJ, A Warped Accretion Disk and Wide-Angle Outflow in the Inner Parsec of the Circinus Galaxy, 590, 162 (2003)

[5] Herrnstein, J. R., Greenhill, L. J., Moran, J. M., ApJL, The Warp in the Subparsec Molecular Disk in NGC4258 as an Explanation for Persistent Asymmetries in the Maser Spectrum, 468, L17 (1996)

[6] Kuijken, K., ApJ, Galactic disk WARPS 376, 467 (1991)

[7] Lodato, G., Pringle, J. E., MNRAS, The evolution of misaligned accretion discs and spinning black holes, 368, 1196 (2006)

[8] Maloney, P. R., Begelman, M. C., Pringle, J. E., ApJ, Radiation-driven Warping: The Origin of WARPS and Precession in Accretion Disks, 472, 582 (1996)

[9] Miyoshi, M., Moran, J., Herrnstein, J. R., Greenhill, L. J., Nakai, N., Diamond, P. \& Inoue, M., Nature, Evidence for a Black-Hole from High Rotation Velocities in a Sub-Parsec Region of NGC4258, 373, 127 (1995)

[10] Natarajan, P., Armitage, P. J., MNRAS, Warped discs and the directional stability of jets in active galactic nuclei, 309, 961 (1999)

[11] Pringle, J.E., MNRAS, Self-induced warping of accretion discs, 281, 357 (1996) 